\title{
The impact of neoadjuvant and adjuvant immunotherapy on the survival of pancreatic cancer patients: a retrospective analysis
}

\author{
Saber Amin ${ }^{1}$, Michael Baine ${ }^{1}$, Jane Meza² and Chi Lin ${ }^{1 *}$ (D)
}

\begin{abstract}
Background: Immunotherapy has become an essential part of cancer treatment after showing great efficacy in various malignancies. However, its effectiveness in pancreatic ductal adenocarcinoma (PDAC), especially in resectable pancreatic cancer, has not been studied. The primary objective of this study is to compare the OS impact of immunotherapy between PDAC patients who receive neoadjuvant immunotherapy and patients who receive adjuvant immunotherapy. The secondary objective is to investigate the impact of neoadjuvant and adjuvant immunotherapy in combination with chemotherapy and chemoradiation by performing subset analyses of these two groups.

Methods: Patients diagnosed with PDAC between 2004 and 2016 were identified from the National Cancer Database (NCDB). Multivariable Cox proportional hazard analysis was performed to examine the effect of neoadjuvant and adjuvant immunotherapy in combination with chemotherapy and chemoradiation on the OS of the patients. The multivariable analysis was adjusted for essential factors such as the age at diagnosis, sex, race, education, income, place of living insurance status, hospital type, comorbidity score, and year of diagnosis.

Results: Overall, 526 patients received immunotherapy. Among whom, 408/526 (77.57\%) received neoadjuvant immunotherapy, and the remaining 118/526 (22.43\%) received adjuvant immunotherapy. There was no significant difference in OS between neoadjuvant and adjuvant immunotherapy (HR: 1.06, Cl: 0.79-1.41; $p<0.714$ ) in the multivariable analysis. In the univariate neoadjuvant treatment subset analysis, immunotherapy was associated with significantly improved OS compared to no immunotherapy (HR: 0.88, Cl: 0.78-0.98; $p<0.026$ ). This benefit disappeared in the multivariable analysis. However, after patients were stratified by educational level, the multivariable Cox regression analysis revealed that neoadjuvant immunotherapy was associated with significantly improved OS (HR: 0.86, Cl: 0.74-0.99; $p<0.04$ ) compared to no immunotherapy only in patients with high-level of education, but not in patients with low-level of education.

(Continued on next page)
\end{abstract}

\footnotetext{
* Correspondence: clin@unmc.edu

${ }^{1}$ Department of Radiation Oncology, University of Nebraska Medical Center,

986861 Nebraska Medical Center, Omaha, NE 68198-6861, USA

Full list of author information is available at the end of the article
}

C C The Author(s). 2020 Open Access This article is licensed under a Creative Commons Attribution 4.0 International License, which permits use, sharing, adaptation, distribution and reproduction in any medium or format, as long as you give appropriate credit to the original author(s) and the source, provide a link to the Creative Commons licence, and indicate if changes were made. The images or other third party material in this article are included in the article's Creative Commons licence, unless indicated otherwise in a credit line to the material. If material is not included in the article's Creative Commons licence and your intended use is not permitted by statutory regulation or exceeds the permitted use, you will need to obtain permission directly from the copyright holder. To view a copy of this licence, visit http://creativecommons.org/licenses/by/4.0/ The Creative Commons Public Domain Dedication waiver (http://creativecommons.org/publicdomain/zero/1.0/) applies to the data made available in this article, unless otherwise stated in a credit line to the data. 
(Continued from previous page)

Conclusion: In this study, no difference in the OS between patients who received neoadjuvant immunotherapy and patients who received adjuvant immunotherapy was noticed. Future studies comparing neoadjuvant adjuvant immunotherapy combined with chemotherapy, radiation therapy, and chemoradiation are needed.

Keywords: Neoadjuvant therapy, Adjuvant therapy, Immunotherapy, Overall survival, Pancreatic adenocarcinoma

\section{Background}

The majority of pancreatic ductal adenocarcinoma (PDAC) patients are diagnosed with unresectable PDAC, while less than $20 \%$ are diagnosed with resectable cancer [1,2]. The current standard-of-care treatment for resectable PDAC is upfront surgery followed by adjuvant single or combined chemotherapy [3]. The median overall survival (OS) after surgery is between 15 and 24 months with a five-year survival rate of $20 \%$ with some recent data showing a median OS of up to 54 months [4-7]. Up to $80 \%$ of patients who undergo surgery experience recurrence, owing significantly to micrometastases, which occur early in the disease, or microscopic residual disease in the tumor bed [1, 2]. These difficulties have brought adjuvant therapy to the forefront of PDAC treatment. In a recent phase III randomized trials, the combination of adjuvant gemcitabine and capecitabine was associated with better median OS (28.0 months, 95\% CI 23.5-31.5 vs. 25.5 months, CI: 22.7-27.9) compared to the gemcitabine alone group (hazard ratio 0.82, CI 0.68-0.98; $p=0.032$ ). The combination of gemcitabine and capecitabine should be the treatment of choice in the adjuvant setting after the surgical resection of adenocarcinoma [8]. Nonetheless, despite improvement in surgical techniques, radiation therapy (RT), and chemotherapeutic options, only a modest improvement in the OS has been noticed [9]. Due to the lack of current standard-of-care treatments, novel treatment strategies such as the use of immunotherapeutics are desperately needed.

Immunotherapy has worked well in many solid cancers, but its use in PDAC is not clear $[10,11]$. The use of immunotherapy to date has been mainly in a metastatic setting. However, new evidence indicates that immunotherapy could be useful in patients with localized disease who have a high risk of micrometastases [12-17]. Chemotherapy and RT increase tumor-specific $\mathrm{T}$ cell infiltration, decrease $\mathrm{T}$ regulatory cells, and suppress Myeloid-derived suppressor cells (MDSCs), and can have synergistic interaction with immunotherapy $[15,18,19]$. Immunotherapy was associated with tumor regression and improved OS in preclinical studies of PDAC when used in combination with other treatments [20, 21]. To achieve the optimal OS effect of the use of immunotherapy with chemotherapy and chemoradiation in PDAC, the sequence of the treatment is critical. The sequence of treatment even becomes more significant in resectable PS due to the potential interactions of systemic therapy with surgery. Due to the higher rate of recurrence after surgery, the early implementation of systemic therapy is needed [22].

Neoadjuvant treatment (NAT) strategies have emerged and been employed as an attractive option for resectable and potentially resectable PDAC [23, 24]. Neoadjuvant treatment can also turn those initially borderline resectable or even some unresectable disease into resectable [23, 24]. This strategy provides an opportunity for an early start of systemic therapy in contrast to upfront surgery, where more than half of the patients may not receive adjuvant therapy due to postoperative complications and declining performance status [25-27]. Recent clinical trials and systematic reviews have reported the survival benefit of NAT [28-31]. However, the effectiveness of NAT in resectable PDAC remains unclear as there are still many questions to be addressed before NAT becomes a standard of care [32].

The neoadjuvant and adjuvant use of immunotherapy both could be justified. Neoadjuvant immunotherapy with chemotherapy or chemoradiation could shrink the tumor, downstage nodal disease, and increase the chance of margin negative resection as reported for neoadjuvant systemic therapy $[33,34]$. It may also work with chemotherapy or chemoradiation to mitigate the risk of micrometastases [35, 36]. Contrary, adjuvant immunotherapy may be effective when the bulk of the tumor is removed, and there is a minimal residual disease, which $\mathrm{T}$ cells can target and eliminate. In addition, the timing of adjuvant immunotherapy needs to be appropriately chosen as surgery is associated with transient immunosuppression $[37,38]$. The use of immunotherapy in neoadjuvant or adjuvant setting combined with chemoradiation in PDAC has been limited. Some clinical trials studying the efficacy of immunotherapy in resectable PDAC combined with chemoradiation therapy have shown positive response and measurable activity [39-42]. However, extensive studies of neoadjuvant and adjuvant immunotherapy in resectable PDAC are lacking. The objective of this study is to investigate the impact of neoadjuvant and adjuvant immunotherapy in combination with chemotherapy and chemoradiation on the OS of resectable PDAC patients using the National Cancer Database (NCDB).

\section{Methods}

Data source

The data for this study were extracted from a deidentified file of the National Cancer Database (NCDB). 
The NCDB is a joint program of the Commission on Cancer of the American College of Surgeons and the American Cancer Society. It captures $70 \%$ or more of newly diagnosed malignancies in the United States annually. This study was exempt from the Institutional Review Board (IRB) because the de-identified data were used.

\section{Study population}

The study included patients age 18 or older who were diagnosed with PADC between 2004 and 2016 and received definitive surgery of the tumor. The ICD-O-3 histology codes of 8000, 8010, 8020-8022, 8140, 8141, $8211,8230,8500,8521,8050,8260,8441,8450,8453$, 8470-8473, 8480, 8481, 8503,8250,8440, 8560 were used to identify PDAC. The surgical site-specific code was used to identify patients with definitive surgery of the pancreas. Patients with missing information related to RT, chemotherapy, immunotherapy, and sequence of these treatments with each other and surgery were excluded. Patients with the M1 stage and those with unknown or missing information about other covariates in the adjusted multivariable analysis were also excluded. The analysis of the sequence of immunotherapy with RT alone was not performed due to the small sample size. The variable of days from diagnosis to the start of the treatment was used to identify neoadjuvant and adjuvant immunotherapy, chemotherapy, and chemoradiation. If chemotherapy, RT, and immunotherapy were delivered more than eight months before or after surgery, those patients were excluded. If immunotherapy was received more than six months before or after chemotherapy or RT, those patients were also excluded. The primary outcome of the current study was the OS of the patients, which was calculated from the date of diagnosis to the date of death. Patients who were alive or lost to follow up were censored. The subset analysis of the neoadjuvant group only included patients who received only neoadjuvant chemotherapy, immunotherapy, RT, and chemoradiation. If any of the treatment was not neoadjuvant, they were excluded from the subset analysis. Patients with no treatment were also excluded. The subset analysis of adjuvant treatment comparison included patients who only received adjuvant chemotherapy, immunotherapy, RT, and chemoradiation. If any of the treatment was not adjuvant, those patients were excluded for adjuvant subset analysis. Patients with no treatment were also excluded from this subset analysis.

\section{Explanatory variables}

The main predictors of OS in this study were immunotherapy combined with chemotherapy, and immunotherapy combined with chemoradiation. The age at diagnosis, gender, race, urban and rural living status, income, education, treatment facility type, comorbidity score, insurance status, year of diagnosis, and receipt of chemotherapy, RT, and immunotherapy were other explanatory variables used in the multivariable analysis.

\section{Statistical analyses}

Descriptive statistics for categorical and continuous variables are reported. A Chi-square test was used to report the association of the explanatory variables with the treatment sequence of immunotherapy with chemotherapy and chemoradiation therapy. The difference in the median OS between the different treatment sequences was reported using the Kaplan-Meier (KM) curves based on the log-ranks test. The Cox regression analysis was used to study the effect of different variables on OS. The estimated hazard ratio (HR) with its associated 95\% confidence intervals (CI) was reported. A p-value of 0.05 was considered significant. The analysis was conducted using the SAS 9.4 software (SAS Enterprise, Cary, NC).

\section{Results \\ Neoadjuvant immunotherapy vs. adjuvant immunotherapy}

Among 526 patients who received immunotherapy, 408/ 526 (77.57\%) received neoadjuvant immunotherapy, and the remaining 118/526 (22.43\%) received adjuvant immunotherapy. The median age at diagnosis among patients who received immunotherapy was 62 with a range of (29-88) years. The median age at diagnosis of patients who received neoadjuvant immunotherapy was 62.0 (34-88) years, while it was $62.5(29-86)$ years in patients who received adjuvant immunotherapy. A majority of the patients were White, living in the urban areas, had a high school degree, had income $>=\$ 35,000$, had insurance, were treated in academic hospitals, and had a Charlson/Deyo Score of zero. There was no association between the baseline characteristics of the patients and receiving neoadjuvant or adjuvant immunotherapy except the year of diagnosis. Among patients who received neoadjuvant immunotherapy, $41.67 \%$ were diagnosed after 2011, while among patients who received adjuvant immunotherapy, 66.10\% were diagnosed after 2011. Among those diagnosed after 2011, 68.55\% received neoadjuvant immunotherapy compared to $31.45 \%$ who received adjuvant immunotherapy, while among those who were diagnosed before 2011, 85.61\% received neoadjuvant immunotherapy compared to $14.39 \%$ who received adjuvant immunotherapy. The baseline characteristics of the study population are shown in Table 1 . We did not report the baseline characteristics of the neoadjuvant and adjuvant subset analyses due to insignificant results of these subsets.

The KM curves based on the log-rank test did not show any significant difference in the median OS of patients who received neoadjuvant immunotherapy compared to 
Table 1 Baseline characteristics of neoadjuvant vs. adjuvant immunotherapy

\begin{tabular}{|c|c|c|c|c|c|}
\hline \multicolumn{2}{|l|}{ Variable } & $\begin{array}{l}\text { Neoadjuvant immunotherapy } \\
408 \text { (77.57\%) }\end{array}$ & $\begin{array}{l}\text { Adjuvant immunotherapy } \\
118(22.43 \%)\end{array}$ & Total 526 & P \\
\hline \multicolumn{2}{|c|}{ Age at diagnosis: median (range) } & $62(34-88)$ & $62.5(29-86)$ & $62.00(29-88)$ & 0.541 \\
\hline \multirow[t]{2}{*}{ Sex } & Male & $238(58.33)$ & $62(52.54)$ & $300(57.03)$ & \\
\hline & Female & $170(41.67)$ & $56(47.46)$ & $226(42.97)$ & 0.263 \\
\hline \multirow[t]{4}{*}{ Race } & White & $359(90.43)$ & $113(96.58)$ & $472(91.83)$ & 0.049 \\
\hline & Black & $21(5.29)$ & $4(3.42)$ & $25(4.86)$ & \\
\hline & Other & $17(4.28)$ & $0(0.00)$ & $17(3.31)$ & \\
\hline & Unknown & 11 & 1 & 12 & \\
\hline \multirow[t]{3}{*}{ Education } & $>=13 \% \mathrm{HG}$ & $112(27.72)$ & $29(24.58)$ & $141(27.01)$ & 0.498 \\
\hline & $<13 \%$ & $292(72.28)$ & $89(75.42)$ & 381 (72.99) & \\
\hline & Unknown & 4 & 0 & 4 & \\
\hline \multirow[t]{3}{*}{ Income } & $>=\$ 35,000$ & $292(72.28)$ & $89(75.42)$ & 381 (72.99) & \\
\hline & $<35,000$ & $112(27.72)$ & $29(24.58)$ & $141(27.01)$ & 0.498 \\
\hline & Unknown & 4 & 0 & 4 & \\
\hline \multirow[t]{3}{*}{ Place of Living } & Urban & $384(98.71)$ & 115 (99.14) & $499(98.81)$ & \\
\hline & Rural & $5(2.29)$ & $1(0.86)$ & $6(1.19)$ & 0.712 \\
\hline & Unknown & 19 & 2 & 21 & \\
\hline \multirow[t]{3}{*}{ Hospital Type } & Academic & $318(78.91)$ & $95(82.61)$ & $413(79.73)$ & \\
\hline & Community & $85(21.09)$ & $20(17.29)$ & $109(20.27)$ & 0.384 \\
\hline & Unknown & 5 & 3 & 8 & \\
\hline \multirow[t]{3}{*}{ Insurance Status } & Insured & $398(98.51)$ & $118(100.00)$ & $516(98.85)$ & \\
\hline & Not insured & $6(1.49)$ & $0(0.00)$ & $6(1.15)$ & 0.183 \\
\hline & Unknown & 4 & 0 & 4 & \\
\hline \multirow[t]{3}{*}{ Charlson/Deyo Score } & 0 & $303(74.26)$ & $88(74.58)$ & $391(74.33)$ & \\
\hline & 1 & $89(21.81)$ & $25(21.19)$ & $114(21.67)$ & \\
\hline & $>=2$ & $16(3.92)$ & $5(4.24)$ & $21(3.99)$ & 0.980 \\
\hline \multirow[t]{2}{*}{ Year of Diagnosis } & 2004-2010 & $238(58.33)$ & $40(33.90)$ & $278(52.85)$ & 0.0001 \\
\hline & $2011-2016$ & $170(41.67)$ & $78(66.10)$ & $248(47.15)$ & \\
\hline
\end{tabular}

adjuvant immunotherapy (Fig. 1). The median OS of patients who received neoadjuvant immunotherapy was 26.78 months (CI: 23.92-31.24) vs. 34.37 months (CI: 24.21-42.28 months; $p=0.703$ ) in patients who received adjuvant immunotherapy. In the multivariable Cox analysis, neoadjuvant immunotherapy was not associated with improved OS (HR: 1.06, CI: $0.79-1.41 ; p=0.714$ ) compared to adjuvant immunotherapy (Table 2).

\section{Subset analyses}

\section{Neoadjuvant subset analysis}

This group was restricted to patients who only received neoadjuvant treatments such as chemotherapy, RT, chemoradiation, and immunotherapy. If any of the treatment was not neoadjuvant, those observations were excluded from this subset analysis. Based on the logrank analysis, patients who received neoadjuvant immunotherapy had significantly improved OS with an absolute median OS benefit of 2.6 months compared to patients who did not receive immunotherapy (25.10 months, CI: $21.42-27.96$ vs. 22.51 months, CI: $22.21-$ 22.77; $p<0.025$ ) (Fig. 2a). In the univariate Cox proportional analysis neoadjuvant immunotherapy was associated with improved OS (HR: 0.88, CI: 0.78-0.98; $p<$ $0.026)$ compared to no immunotherapy. However, in the multivariable analysis, this association became nonsignificant (Table 3). In order to find the explanatory factor in the multivariate analysis, which was responsible for the loss of significance in the effect of immunotherapy on OS, we performed stepwise regression analysis to determine the factor(s) which may interact with neoadjuvant immunotherapy. In the stepwise regression analysis, age at diagnosis, income, education, and hospital type showed interaction with immunotherapy, and the effect of immunotherapy on OS became insignificant when any of these variables were added to the model with immunotherapy. We then stratified by the categories of each of these variables and found that immunotherapy 


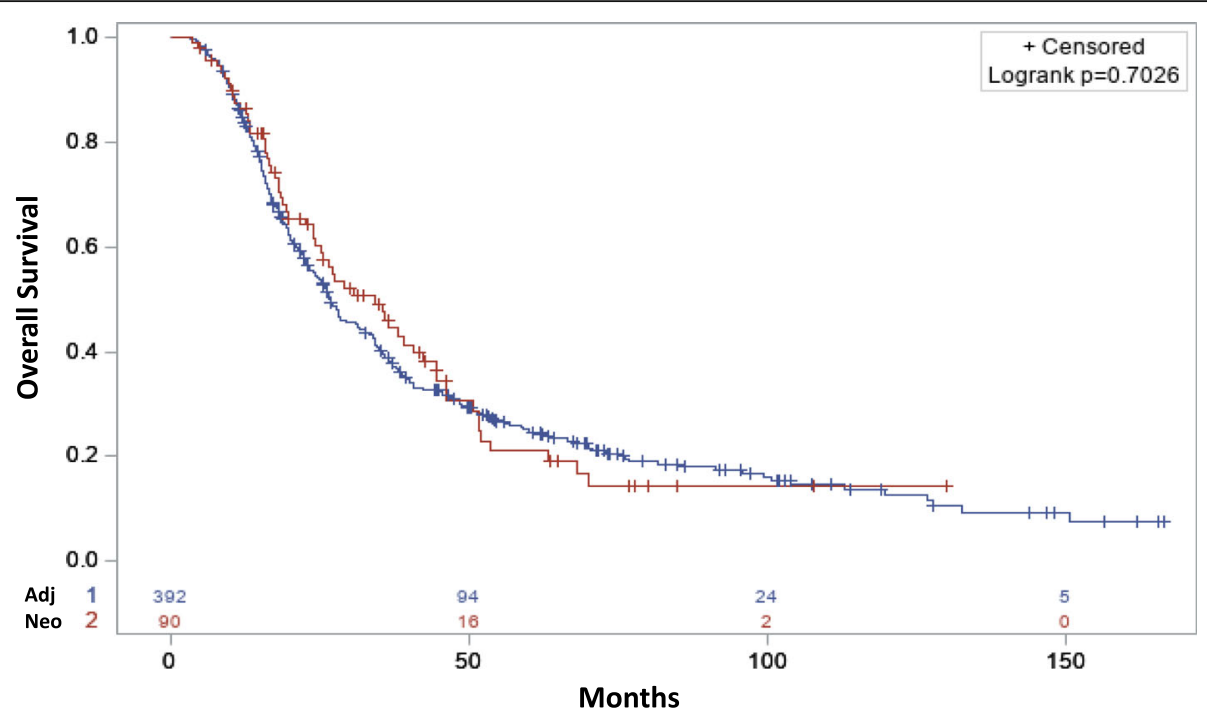

Fig. 1 Overall survival neoadjuvant (red) vs. adjuvant (blue) immunotherapy for the entire cohort

only stayed significant in patients who were living in areas with $<13 \%$ people with no high school degree. After patients were stratified by educational level, we found that in patients who live in areas with a high level of education, the impact of immunotherapy on OS stayed significant despite income, age at diagnosis, and hospital type. In the multivariable Cox regression analysis, neoadjuvant immunotherapy was associated with significantly improved OS (HR: 0.86, CI: 0.74-0.99; $p<0.04$ ) compared to no immunotherapy only in patients with high-level of education, but not in patients with low-level of education.

Further analysis revealed that there was no difference in the median OS of patients who received neoadjuvant chemotherapy plus immunotherapy compared to neoadjuvant chemotherapy alone (Fig. 2b) and patients who received neoadjuvant chemoradiation plus immunotherapy compared patients who received only neoadjuvant chemoradiation (Fig. 2c). In the multivariable analysis, there was no difference in the OS of patients who received neoadjuvant chemotherapy plus immunotherapy compared to neoadjuvant chemotherapy alone (HR: 0.930 , CI: $0.725-1.195 ; p=0.972$ ) and patients who received neoadjuvant chemoradiation plus immunotherapy compared to neoadjuvant chemoradiation alone (HR: 0.942, CI: 0.814-1.091; $p=0.425$ ) (Table 3).

\section{Adjuvant subset analysis}

This analysis included patients who only received adjuvant chemotherapy, RT, chemoradiation, and immunotherapy. If any of the treatment was not adjuvant those patients were not included in this subset analysis. Based on KM curves there was no difference in the median OS of patients who received adjuvant immunotherapy compared to patients who received other adjuvant treatment but did not receive immunotherapy (Fig. 3a). There was no difference in the median OS of patients received adjuvant chemotherapy plus immunotherapy or chemoradiation plus immunotherapy compared chemotherapy or chemoradiation without immunotherapy (Fig. 3b). In the multivariable analysis, there was no significant difference in the OS of patients who received adjuvant immunotherapy compared to no immunotherapy (HR:1.00, CI: $0.76-1.32 ; p=0.995)$. A significant difference in the OS was also not observed between patients who received adjuvant chemotherapy plus immunotherapy or chemoradiation plus immunotherapy compared chemotherapy or chemoradiation without immunotherapy (HR: 1.01, CI: 0.75-1.37; $p=0.935$ ) (Table 4). The adjuvant chemotherapy plus immunotherapy group was combined with chemoradiation plus immunotherapy due to a small sample size.

Table 2 Univariate and multivariate Cox regression analysis of neoadjuvant immunotherapy vs. adjuvant immunotherapy

\begin{tabular}{|c|c|c|c|c|c|c|}
\hline \multirow[t]{2}{*}{ Variable } & & \multirow[t]{2}{*}{ N (\%) } & \multicolumn{2}{|l|}{ Univariable analysis } & \multicolumn{2}{|l|}{ Multivariable analysis } \\
\hline & & & Hazard Ratio $(95 \% \mathrm{Cl})$ & $P$ & Hazard Ratio (95\% Cl) & $P$ \\
\hline \multirow[t]{2}{*}{ Immunotherapy } & Neoadjuvant immunotherapy & $408(77.57)$ & $1.06(0.80-1.39)$ & 0.703 & $1.06(0.79-1.41)$ & 0.714 \\
\hline & Adjuvant immunotherapy & $118(22.43)$ & Ref & & Ref & \\
\hline
\end{tabular}

The multivariable analysis was adjusted for the age at diagnosis, sex, race, income, education, place of living, treatment facility type, insurance status, comorbidity score, and year of diagnosis 

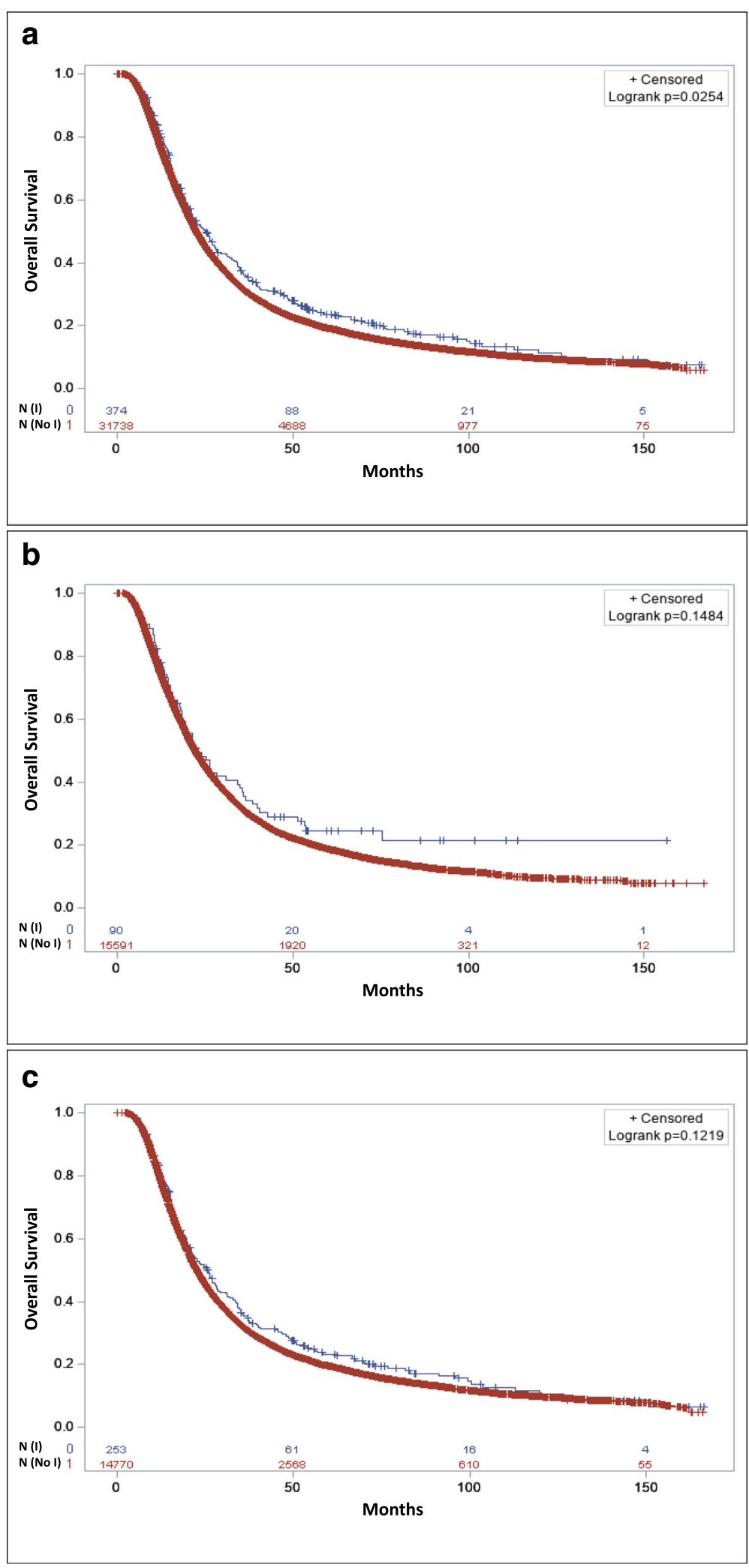

Fig. 2 Overall survival with (blue) or without (red) neoadjuvant immunotherapy for (a) all patients with only neoadjuvant therapies; b patients who received neoadjuvant chemotherapy; $\mathbf{c}$ patients who received neoadjuvant chemoradiation 
Table 3 Cox regression analysis of only neoadjuvant immunotherapy combinations

\begin{tabular}{|c|c|c|c|c|c|c|}
\hline \multirow[t]{2}{*}{ Variable } & & \multirow[t]{2}{*}{ N (\%) } & \multicolumn{2}{|l|}{ Univariable analysis } & \multicolumn{2}{|l|}{ Multivariable analysis } \\
\hline & & & Hazard Ratio (95\% Cl) & $P$ & Hazard Ratio $(95 \% \mathrm{Cl})$ & $\mathrm{P}$ \\
\hline \multirow[t]{2}{*}{ Immunotherapy } & Neoadjuvant immunotherapy & $373(1.09)$ & $0.88(0.78-0.98)$ & 0.026 & $0.93(0.82-1.05)$ & 0.220 \\
\hline & No immunotherapy & $33,921(98.91)$ & Ref & & Ref & \\
\hline \multirow[t]{2}{*}{ CTx plus immunotherapy } & Neoadjuvant CTx plus imm & $95(0.53)$ & $0.835(0.653-1.067)$ & 0.150 & $0.930(0.725-1.195)$ & 0.572 \\
\hline & Adjuvant CTx only & $17,868(99.47)$ & Ref & & & \\
\hline \multirow{2}{*}{ CTXRT plus immunotherapy } & Neoadjuvant CTxRTx plus imm & $258(1.64)$ & $0.897(0.781-1.030)$ & 0.122 & $0.942(0.814-1.091)$ & 0.425 \\
\hline & Adjuvant CTxRTx only & $15,466(98.36)$ & Ref & & & \\
\hline
\end{tabular}

CTx: chemotherapy, CTxRTx: chemoradiation therapy, imm: immunotherapy

\section{Discussion}

To our knowledge, the current study is the most extensive study that has compared the impact of neoadjuvant immunotherapy vs. adjuvant immunotherapy on the OS of PDAC patients who received definitive surgery of the pancreatic tumor. There was no significant difference in the median OS of patients who received neoadjuvant immunotherapy compared to patients who received adjuvant immunotherapy. However, in the neoadjuvant subset analysis, immunotherapy was associated with significantly improved OS compared to no immunotherapy in the univariate analysis though this significance was lost upon multivariable analysis.

The improved OS of neoadjuvant immunotherapy compared to no immunotherapy in the univariate analysis may be due to the impact of immunotherapy in eradicating the occult micrometastases that occur early in PDAC $[1,2]$. The tumor cells use mechanisms such as the up-regulation of immune checkpoint signaling programmed death-ligand 1 (PD-L1), downregulation of cytotoxic T-lymphocyte-associated protein 4 (CTLA4), and the recruitment of MDSCs, to evade the immune system [43-45]. Immunotherapy, especially checkpoint inhibitors, down-regulates the PD-L1 pathway and upregulates anti-CTLA4 $[16,19]$. The insignificant results of neoadjuvant immunotherapy compared to adjuvant immunotherapy may indicate that the impact of immunotherapy on the OS of PDAC patients who receive definitive surgery of the pancreatic tumor is not related to the sequence of immunotherapy with surgery. A small sample size of group comparisons in the neoadjuvant and adjuvant subsets analyses may be responsible for insignificant results. Neoadjuvant chemotherapy plus immunotherapy was not associated with improved OS compared to neoadjuvant chemotherapy. However, the extended plateaued or nearly a flat line at the end of the $\mathrm{KM}$ curve is indicative of the long-lasting immunity or cure from cancer, which is only seen in patients who received both chemotherapy and immunotherapy (Fig. 2b, blue). This is strong evidence of long-lasting immunity and possible cure from cancer in patients who received neoadjuvant chemotherapy plus immunotherapy.
The year of diagnosis could interact with both chemotherapy and immunotherapy. Treatment with chemotherapy and immunotherapy has evolved over the years and will likely influence the OS of the resectable pancreatic cancer patients. The 5-year survival rate after resection improved to $16-21 \%$ in pancreatic patients who receive adjuvant chemotherapy with gemcitabine or 5fluorouracil plus folinic acid after resection compared to $10 \%$ for surgery alone [8]. Median survival time of up to 54 months has been reported with adjuvant modified FOLFIRINOX in resected pancreatic cancer patients [4]. Checkpoint inhibitors, the most widely used immunotherapy, became available only after 2013. Patients who were diagnosed after 2013 are likely to have received a checkpoint inhibitor and may have better OS compared to patients who either received other types of immunotherapy or were diagnosed before 2013. We performed separate multivariable analyses for patients who were diagnosed after 2013 and patients who were diagnosed before 2013. In both stratified analyses, there was no difference in the OS of patients who received neoadjuvant immunotherapy and patients who received adjuvant immunotherapy. There was not enough sample size to stratify by individual year.

\section{Limitations}

The large sample size for the Comparison of neoadjuvant immunotherapy vs. adjuvant immunotherapy is the most important strength of the current study, which allowed us to adjust for patient and tumor characteristics. However, the study is not without limitations, most of which are inherent to NCDB and include selections bias, lack of information of the cause of death, lack of information about the type of immunotherapy and if a single or combined immunotherapy was administered, and lack of detailed information on the use of multi-agent chemotherapy. Another limitation is the inability to calculate progression-free survival as the NCDB does not provide information on disease progression or recurrence. One other limitation was that due to the small sample size for immunotherapy plus RT, the sequence of immunotherapy with RT alone was not performed. In 


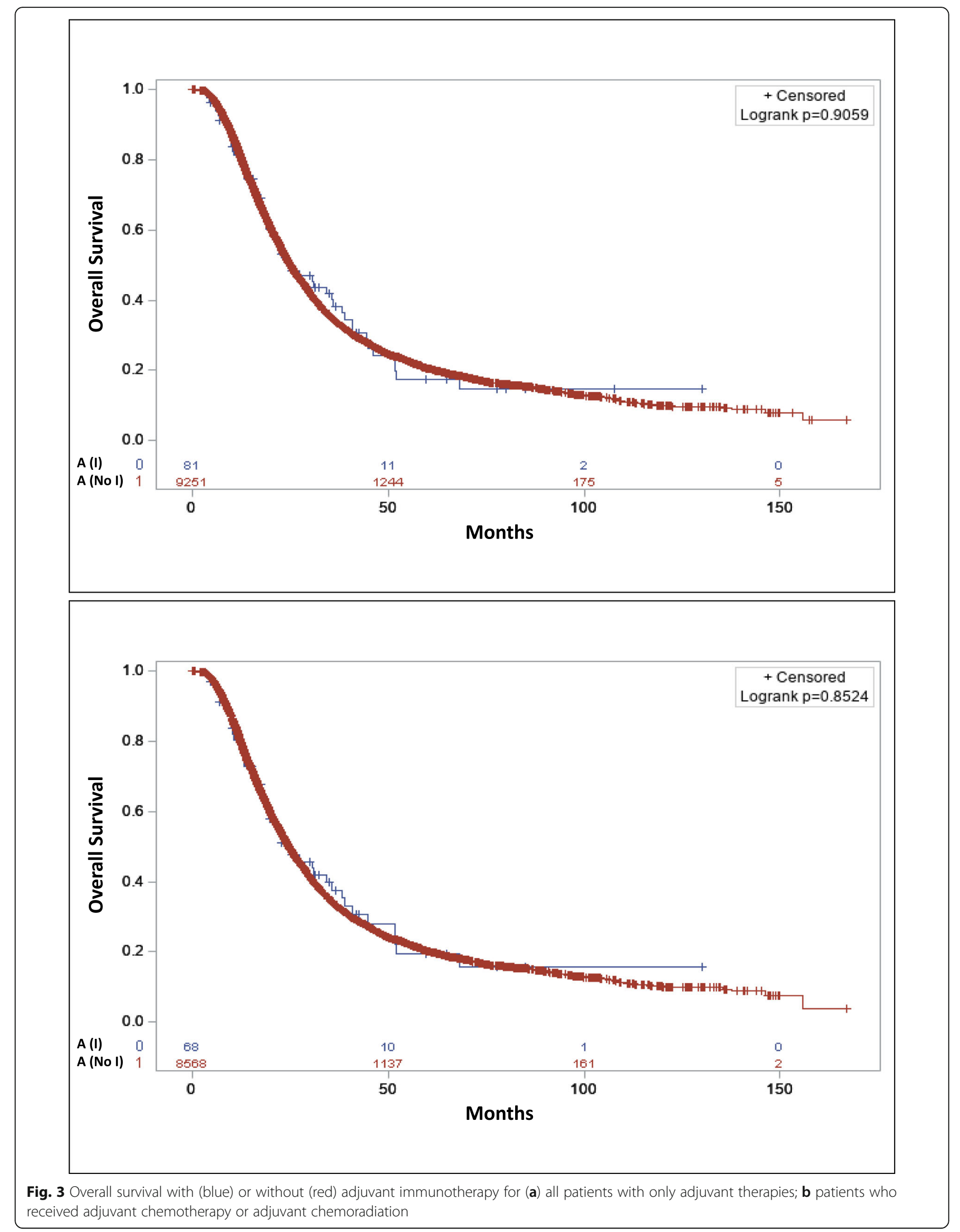


Table 4 Cox regression analysis of only adjuvant immunotherapy combinations

\begin{tabular}{|c|c|c|c|c|c|c|}
\hline \multirow[t]{2}{*}{ Variable } & & \multirow[t]{2}{*}{ N (\%) } & \multicolumn{2}{|l|}{ Univariable analysis } & \multicolumn{2}{|l|}{ Multivariable analysis } \\
\hline & & & Hazard Ratio (95\% Cl) & $P$ & Hazard Ratio (95\% Cl) & $\mathrm{P}$ \\
\hline \multirow[t]{2}{*}{ Immunotherapy } & Adjuvant immunotherapy & $106(0.96)$ & $0.98(0.76-1.28)$ & 0.907 & $1.00(0.76-1.32)$ & 0.995 \\
\hline & No immunotherapy & $10,950(99.04)$ & Ref & & Ref & \\
\hline \multirow[t]{2}{*}{ CTx or CTxRTx plus immunotherapy } & Adjuvant CTx or CTxRTx plus imm & $90(0.88)$ & $0.97(0.73-1.30)$ & 0.854 & $1.01(0.75-1.37)$ & 0.935 \\
\hline & Adjuvant CTx or CTxRTx & $10,104(99.12)$ & Ref & & Ref & \\
\hline
\end{tabular}

We combined adjuvant chemotherapy plus immunotherapy with adjuvant chemoradiation plus immunotherapy due to small sample size. When analyzed separately the results were the same. CTx: chemotherapy, CTxRTx: chemoradiation therapy, imm: immunotherapy

addition, there were not enough cases for adjuvant comparison, and that maybe one of the reasons that we failed to find any significant difference in the OS of patients who received adjuvant immunotherapy, chemotherapy plus immunotherapy, and chemoradiation plus immunotherapy compared to their counterparts without immunotherapy.

Nonetheless, in this study, a robust analysis of the impact of the timing of immunotherapy with surgery on the OS of PDAC patients who received definitive surgery of the pancreatic tumor using the NCDB was performed. The NCDB is the largest cancer database in the world which captures the majority of the annual cancer cases diagnosed in the U.S. It serves as an outstanding source for the investigation of the impact of novel cancer treatments on the OS of cancer patients.

\section{Conclusions}

No difference in the OS between patients who received neoadjuvant immunotherapy and those who receive adjuvant immunotherapy was noticed. However, in the univariate analysis, neoadjuvant immunotherapy was associated with significantly improved OS compared to no immunotherapy. The findings warrant future studies with a large sample size for both neoadjuvant and adjuvant treatment comparisons of immunotherapy.


adenocarcinoma; MDSCs: Myeloid-derived suppressor cells; RT: Radiation therapy; PD-L1: Programmed death-ligand 1; CTLA4: Cytotoxic T-lymphocyteassociated protein 4; CTx: Chemotherapy; Imm: Immunotherapy;

CTxRTx: Chemoradiation therapy; RTx: Radiation therapy

\section{Acknowledgments}

Not applicable.

\section{Disclosure statement}

The authors have no conflicts of interest to disclose. There are no acknowledgments. There was no funding for this study. This study has not been presented or published in part or full form elsewhere.

\section{Authors' contributions}

SA: Design, Analysis, manuscript writing, review, and editing; MB: Design, review, and editing; JM: Analysis, review, and editing; CL: Design, writing, review, and editing. All authors read and approved the final version of the manuscript.
Funding

Not applicable.

\section{Availability of data and materials}

The datasets used and analyzed during the current study are available from the corresponding author on reasonable request.

\section{Ethics approval and consent to participate}

This study used data from the NCDB. The data is de-identified and holds no identifying patient information, and therefore, written informed consent was not needed for this study. The institutional review board (IRB) was also not required for the study.

\section{Consent for publication}

Not applicable.

\section{Competing interests}

The authors declare that they have no competing interests.

\section{Author details}

'Department of Radiation Oncology, University of Nebraska Medical Center, 986861 Nebraska Medical Center, Omaha, NE 68198-6861, USA. ${ }^{2}$ Department of Biostatistics, College of Public Health, University of Nebraska Medical Center, Omaha, USA.

Received: 20 April 2020 Accepted: 28 May 2020

Published online: 09 June 2020

\section{References}

1. Siegel RL, Miller KD, Jemal A. Cancer statistics, 2017. CA Cancer J Clin. 2017; 67(1):7-30.

2. $\quad$ Li D, Xie K, Wolff R, Abbruzzese JL. Pancreatic cancer. Lancet. 2004; 363(9414):1049-57.

3. Seufferlein T, Ettrich TJ. Treatment of pancreatic cancer-neoadjuvant treatment in resectable pancreatic cancer (PDAC). Transl Gastroenterol Hepatol. 2019;4:21.

4. Conroy T, Hammel P, Hebbar M, et al. FOLFIRINOX or gemcitabine as adjuvant therapy for pancreatic cancer. N Engl J Med. 2018;379(25):2395406.

5. Yeo CJ, Abrams RA, Grochow LB, et al. Pancreaticoduodenectomy for pancreatic adenocarcinoma: postoperative adjuvant chemoradiation improves survival. A prospective, single-institution experience. Ann Surg. 1997;225(5):621-33 discussion 633-6.

6. Yeo CJ, Cameron JL, Lillemoe KD, et al. Pancreaticoduodenectomy for cancer of the head of the pancreas. 201 patients. Ann Surg. 1995;221(6): 721-31 discussion 731-3.

7. Neoptolemos JP, Stocken DD, Bassi C, et al. Adjuvant chemotherapy with fluorouracil plus folinic acid vs gemcitabine following pancreatic cancer resection: a randomized controlled trial. JAMA. 2010;304(10):1073-81.

8. Neoptolemos JP, Palmer DH, Ghaneh P, et al. Comparison of adjuvant gemcitabine and capecitabine with gemcitabine monotherapy in patients with resected pancreatic cancer (ESPAC-4): a multicentre, open-label, randomised, phase 3 trial. Lancet. 2017;389(10073):1011-24.

9. Lopez NE, Prendergast C, Lowy AM. Borderline resectable pancreatic cancer: definitions and management. World J Gastroenterol. 2014;20(31):10740-51.

10. Menon S, Shin S, Dy G. Advances in cancer immunotherapy in solid tumors. Cancers (Basel). 2016;8(12). https://doi.org/10.3390/cancers8120106. 
11. Hilmi M, Bartholin $L$, Neuzillet C. Immune therapies in pancreatic ductal adenocarcinoma: where are we now? World J Gastroenterol. 2018;24(20): 2137-51.

12. Markowitz GJ, Havel LS, Crowley MJ, et al. Immune reprogramming via PD-1 inhibition enhances early-stage lung cancer survival. JCI Insight. 2018;3(13). https://doi.org/10.1172/jci.insight.96836.

13. Chiari R, Sidoni A, Metro G. Early stage resectable non-small cell lung cancer: is neoadjuvant immunotherapy the right way forward? J Thorac Dis. 2018;10(Suppl 33):S3890-4.

14. Ghysen K, Vansteenkiste J. Immunotherapy in patients with early stage resectable nonsmall cell lung cancer. Curr Opin Oncol. 2019;31(1):13-7.

15. Young K, Hughes DJ, Cunningham D, Starling N. Immunotherapy and pancreatic cancer: unique challenges and potential opportunities. Ther Adv Med Oncol. 2018;10:1758835918816281.

16. Rosenberg A, Mahalingam D. Immunotherapy in pancreatic adenocarcinoma-overcoming barriers to response. J Gastrointest Oncol. 2018;9(1):143-59.

17. Forde PM, Chaft JE, Smith KN, et al. Neoadjuvant PD-1 blockade in resectable lung cancer. N Engl J Med. 2018;378(21):1976-86.

18. Chang $J H$, Jiang $Y$, Pillarisetty VG. Role of immune cells in pancreatic cancer from bench to clinical application: an updated review. Medicine (Baltimore). 2016;95(49):e5541.

19. Torphy RJ, Zhu Y, Schulick RD. Immunotherapy for pancreatic cancer: barriers and breakthroughs. Ann Gastroenterol Surg. 2018;2(4):274-81.

20. Beatty GL, Chiorean EG, Fishman MP, et al. CD40 agonists alter tumor stroma and show efficacy against pancreatic carcinoma in mice and humans. Science. 2011;331(6024):1612-6.

21. Byrne KT, Vonderheide RH. CD40 stimulation obviates innate sensors and drives T cell immunity in cancer. Cell Rep. 2016;15(12):2719-32.

22. Roth MT, Berlin JD. Current concepts in the treatment of resectable pancreatic cancer. Curr Oncol Rep. 2018;20(5):39-018-0685-y.

23. Evans DB, Varadhachary GR, Crane $\mathrm{CH}$, et al. Preoperative gemcitabinebased chemoradiation for patients with resectable adenocarcinoma of the pancreatic head. J Clin Oncol. 2008;26(21):3496-502.

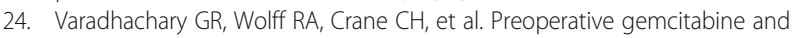
cisplatin followed by gemcitabine-based chemoradiation for resectable adenocarcinoma of the pancreatic head. J Clin Oncol. 2008;26(21):3487-95.

25. Herman JM, Swartz MJ, Hsu CC, et al. Analysis of fluorouracil-based adjuvant chemotherapy and radiation after pancreaticoduodenectomy for ductal adenocarcinoma of the pancreas: results of a large, prospectively collected database at the johns Hopkins hospital. J Clin Oncol. 2008;26(21):3503-10.

26. Corsini MM. Adjuvant radiotherapy and chemotherapy for pancreatic carcinoma: The mayo clinic experience (1975-2005). J Clin Oncol. 26(21): 3511-6.

27. Parmar AD, Vargas GM, Tamirisa NP, Sheffield KM, Riall TS. Trajectory of care and use of multimodality therapy in older patients with pancreatic adenocarcinoma. Surgery. 2014;156(2):280-9.

28. Versteijne E, Suker M, Groothuis K, et al. Preoperative chemoradiotherapy versus immediate surgery for resectable and borderline resectable pancreatic cancer: Results of the dutch randomized phase III PREOPANC trial. J Clin Oncol. 2020:JCO1902274.

29. Motoi F, Kosuge $T$, Ueno $H$, et al. Randomized phase II/III trial of neoadjuvant chemotherapy with gemcitabine and S-1 versus upfront surgery for resectable pancreatic cancer (prep-02/JSAP05). Jpn J Clin Oncol. 2019;49(2):190-4.

30. de Geus SW, Evans DB, Bliss LA, et al. Neoadjuvant therapy versus upfront surgical strategies in resectable pancreatic cancer: a markov decision analysis. Eur J Surg Oncol. 2016;42(10):1552-60.

31. Mokdad AA, Minter RM, Zhu H, et al. Neoadjuvant therapy followed by resection versus upfront resection for resectable pancreatic cancer: a propensity score matched analysis. J Clin Oncol. 2017;35(5):515-22.

32. Dhir M, Malhotra GK, Sohal DPS, et al. Neoadjuvant treatment of pancreatic adenocarcinoma: a systematic review and meta-analysis of 5520 patients. World J Surg Oncol. 2017;15(1):183 -017-1240-2.

33. Tsai S, Christians KK, Ritch PS, et al. Multimodality therapy in patients with borderline resectable or locally advanced pancreatic cancer: importance of locoregional therapies for a systemic disease. J Oncol Pract. 2016;12(10): 915-23.

34. Sohal DP, Walsh RM, Ramanathan RK, Khorana AA. Pancreatic adenocarcinoma: Treating a systemic disease with systemic therapy. J Nat Cancer Inst. 2014;106(3):dju011.
35. Lee YS, Lee JC, Yang SY, Kim J, Hwang JH. Neoadjuvant therapy versus upfront surgery in resectable pancreatic cancer according to intention-totreat and per-protocol analysis: a systematic review and meta-analysis. Sci Rep. 2019;9(1):15662 -019-52167-9.

36. Wolff RA. Adjuvant or neoadjuvant therapy in the treatment in pancreatic malignancies: Where are we? Surg Clin North Am. 2018;98(1):95-111. https://doi.org/10.1016/j.suc.2017.09.009.

37. Lennard TW, Shenton BK, Borzotta A, et al. The influence of surgical operations on components of the human immune system. Br J Surg. 1985; 72(10):771-6.

38. Zhang P, Cote AL, de Vries VC, Usherwood EJ, Turk MJ. Induction of postsurgical tumor immunity and T-cell memory by a poorly immunogenic tumor. Cancer Res. 2007;67(13):6468-76.

39. Katz MHG, Varadhachary GR, Bauer TW, et al. Preliminary safety data from a randomized multicenter phase ib/ll study of neoadjuvant chemoradiation therapy (CRT) alone or in combination with pembrolizumab in patients with resectable or borderline resectable pancreatic cancer. JCO. 2017;35(15):4125.

40. Lutz $E$, Yeo CJ, Lillemoe KD, et al. A lethally irradiated allogeneic granulocyte-macrophage colony stimulating factor-secreting tumor vaccine for pancreatic adenocarcinoma. A phase II trial of safety, efficacy, and immune activation. Ann Surg. 2011;253(2):328-35.

41. Aguilar LK, Shirley LA, Chung VM, et al. Gene-mediated cytotoxic immunotherapy as adjuvant to surgery or chemoradiation for pancreatic adenocarcinoma. Cancer Immunol Immunother. 2015;64(6):727-36.

42. Hardacre JM, Mulcahy M, Small W, et al. Addition of algenpantucel-L immunotherapy to standard adjuvant therapy for pancreatic cancer: A phase 2 study. J Gastrointest Surg. 2013;17(1):94-100 discussion p. 100-1.

43. Chanmee T, Ontong P, Konno K, Itano N. Tumor-associated macrophages as major players in the tumor microenvironment. Cancers (Basel). 2014;6(3): 1670-90.

44. Ohaegbulam KC, Assal A, Lazar-Molnar E, Yao Y, Zang X. Human cancer immunotherapy with antibodies to the PD-1 and PD-L1 pathway. Trends Mol Med. 2015;21(1):24-33.

45. Umansky V, Sevko A. Tumor microenvironment and myeloid-derived suppressor cells. Cancer Microenviron. 2013;6(2):169-77.

\section{Publisher's Note}

Springer Nature remains neutral with regard to jurisdictional claims in published maps and institutional affiliations.

Ready to submit your research? Choose BMC and benefit from:

- fast, convenient online submission

- thorough peer review by experienced researchers in your field

- rapid publication on acceptance

- support for research data, including large and complex data types

- gold Open Access which fosters wider collaboration and increased citations

- maximum visibility for your research: over $100 \mathrm{M}$ website views per year

At BMC, research is always in progress.

Learn more biomedcentral.com/submissions 